\title{
Percepción de los profesores de la Licenciatura de Comunicación y Periodismo de la Universidad Autónoma de Querétaro (UAQ), México, sobre la interacción y el uso de herramientas TIC para colaborar
}

\author{
Carlos Alberto Rode Villa ${ }^{1 *}$
}

\begin{abstract}
Resumen
El objetivo del trabajo que se reporta fue conocer cómo se da la interacción en el aprendizaje colaborativo en alumnos de la Licenciatura en Comunicación y Periodismo de la Universidad Autónoma de Querétaro. Utilizando entrevistas semiestructuradas aplicadas a profesores, se indagó sobre dos dimensiones de colaboración: la interacción y el uso de herramientas tecnológicas. Se encuentra que no hay una verdadera interacción en el trabajo colaborativo, por problemas al trabajar, ya que existen integrantes que aportan y otros que no. Se utilizan dos herramientas para la comunicación: Facebook y WhatsApp. La interacción no se logra al máximo y la tecnología no es vital para trabajar en conjunto.

\section{Abstract}

The objective of this report was to know how the interaction in collaborative learning occurs in the students in Communication and Journalism of the Autonomous University of Queretaro. Through semi-structured interviews, professors were inquired about two dimensions of the collaboration: the interaction and the use of technological tools. It was found that there is no real interaction in collaborative work, some causes are: working problems with partners; and that there are two types of members; those who work and those who do not. Two tools are used for communication: Facebook and WhatsApp. The interaction is not achieved to the maximum in this process and the technology is not vital to work together.
\end{abstract}

\section{Palabras Clave}

Aprendizaje, Colaboración, Interacción, TIC

Keywords

Learning, Collaboration; Interaction, ICT

${ }^{1}$ Maestro en Comunicación y Tecnología Educativa. Universidad Autónoma de Querétaro, México.

*Autor para correspondencia: crode69@hotmail.com

\section{Introducción}

Este artículo es parte de una investigación en proceso que se lleva a cabo para obtener el grado de doctorado en Tecnología Educativa, en la que se pretende realizar una estrategia colaborativa mediada por tecnología para alumnos de la Licenciatura de Comunicación y Periodismo de la Universidad Autónoma de Querétaro.
La información recabada forma parte del diagnóstico para conocer las prácticas del trabajo colaborativo existentes en clases donde los profesores trabajan por proyectos y en equipo en la Facultad de Ciencias Políticas y Sociales.

La interacción es uno de los elementos necesarios para que se dé el aprendizaje colaborativo, cuando los alumnos trabajan de forma conjunta generan distintos procesos y relaciones interpersonales donde aprenden de sus compañeros, ya que este tipo de relaciones les permite escuchar y ser escuchados, 
intercambiar y negociar información en la búsqueda de nuevos conocimientos, entre más formas tengan los alumnos de interactuar, más pueden aprender unos de los otros.

Si bien, la interacción no es el único criterio para que exista un verdadero trabajo colaborativo, sí es uno a los que se le debe dar importancia, ya que la calidad de la interacción permite conocer si en verdad los alumnos están aprendiendo entre ellos, o si solo se relacionan lo necesario para cumplir con la meta académica que en muchos de los casos podría ser alcanzada de forma individual. Por lo que en el presente estudio se buscó estar al tanto sobre el cómo perciben los profesores la interacción entre sus alumnos y qué relevancia le atribuyen a las redes sociales en este proceso. Conocer esto permitirá saber qué tan cerca están los equipos de esta licenciatura en trabajar de forma colaborativa o si bien trabajan solamente como grupos tradicionales.

\section{Marco teórico}

Los estudiantes durante mucho tiempo han trabajado actividades de sus clases de manera grupal: investigan, producen información y realizan prácticas juntos. Este tipo de labores, ¿necesariamente fomentan el aprendizaje colaborativo? o ¿qué características debe tener una actividad para considerarse que existe trabajo colaborativo? La colaboración en grupo es un componente esencial en la educación actual. Los procesos de enseñanza-aprendizaje y sus actividades requieren utilizar métodos donde el alumno desarrolle su capacidad para aprender y comprender a los demás y en este proceso pueda aprender de sus propias ideas, desarrollando una capacidad de interpretación de las ideas de otros, más allá de solo aprender conceptos (García \& García en González, González, de la Cruz, Fuentes, Aguirre \& González, 2012).

Para Johnson, Johnson y Smith (1998), en el trabajo cooperativo debe existir una meta común que se comparta por los miembros del equipo, los resultados de esta actividad académica deben de beneficiar de forma personal y grupal a los alumnos, este tipo de aprendizaje maximiza el aprendizaje individual y grupal. Stahl, Koschman y Suther (2006) hablan de la colaboración como un proceso en el cual los individuos negocian y comparten significados que les son relevantes para solucionar una tarea o problema. La colaboración es, en sus palabras, una actividad coordinada, sincrónica, que es el resultado de un intento continuo de construir y mantener una concepción compartida de un problema.

Es importante entender que el aprendizaje colaborativo se puede dar de diversas formas en el salón de clases. Una forma específica de aprendizaje colaborativo es el mediado por una computadora y, más allá de esto, el que se da en computadoras que están conectadas en redes, y que permiten la comunicación de los estudiantes sin importar las barreras del tiempo y el espacio. El aprendizaje colaborativo apoyado por computadora (CSL, por sus siglas en inglés) es, según Stahl et al. (2006), "un área emergente de las ciencias del aprendizaje referente a estudiar cómo las personas pueden aprender de manera conjunta con la ayuda de los computadores" (p.1). La diferencia entre este tipo de aprendizaje colaborativo soportado por computadora y el presencial, es la incorporación de las redes virtuales y la informática en estos espacios. Lucero (2003) lo define como:

El conjunto de métodos de instrucción y entrenamiento apoyados con tecnología, así como de estrategias para propiciar el desarrollo de habilidades mixtas (aprendizaje, desarrollo personal y social), donde cada miembro del grupo es responsable tanto de su aprendizaje como del de los restantes miembros del grupo (p.4).

El papel que juegan las Tecnologías de la Información y la Comunicación (TIC) con sus redes computacionales en el aprendizaje colaborativo es cada vez más determinante en proyectos educativos presenciales, semipresenciales o en línea, ya que las TIC son mediadoras que potencian el proceso de enseñanza aprendizaje. El aprendizaje colaborativo es un proceso donde dos o más sujetos se unen para llevarlo a cabo, por medio de la discusión, reflexión y las decisiones que deben tomar. En este caso las redes computacionales actúan como mediadores psicológicos para eliminar las barreras espacio-tiempo. Lo que se busca es que los participantes trabajen en proyectos que les interesen a ambos o a todos y que de esta manera se llegue a la resolución de problemas a través de la toma de decisiones en conjunto (Díaz Barriga \& Morales 2008, p.6).

Las TIC y sus redes computacionales son herramientas que dan ventaja ya que permiten estimular: la comunicación 
interpersonal; el acceso a la información y contenidos de aprendizaje; el seguimiento del progreso del participante (individual y grupal); la gestión y administración de alumnos; la creación de escenarios de evaluación (Díaz Barriga \& Morales 2008, p.6). La tabla 1 muestra las distintas herramientas TIC para el aprendizaje colaborativo recuperadas por Colvin y Meyer (2008, citados en Díaz Barriga \& Morales, 2008; Vinagre, 2010).

Las herramientas se dividen según su uso:

1. Comunicación: permiten que la comunicación se realice de forma síncrona o asíncrona y su uso lleva a apoyar las discusiones grupales para intercambiar información, resolver problemas o definir formas de trabajo.

2. Trabajo en la red: brindan la oportunidad de producir $\mathrm{y}$ publicar el material que generan los alumnos y compartirlo en la red, además permiten a los estudiantes aportar su opinión sobre los trabajos de sus compañeros escribiéndoles comentarios.

3. Herramientas que facilitan el trabajo colaborativo y su gestión: sirven para que los estudiantes trabajen compartiendo los mismos documentos y recursos, además de comunicarse de forma asíncrona o síncrona, les permite gestionar tiempos y verificar los avances de los miembros de cada equipo.

4. De investigación: entre estas se encuentran los buscadores (Google, Firefox, etc.) y los agregadores (Greatnews, Feedreader, etc.) que posibilitan al alumno buscar la información necesaria para la resolución de los problemas planteados en un ambiente de aprendizaje colaborativo.

En el caso de esta investigación se dio preferencia a conocer qué tipo de herramientas de las TIC usan los profesores y los alumnos para interactuar cuando no están conviviendo en forma presencial y se encuentran realizando trabajo en equipo o de forma colaborativa. También se averiguó sobre el uso que se les da de forma específica a este tipo de herramientas tecnológicas, para conocer si realmente son usadas y aprovechadas por alumnos y profesores. Una vez definido lo que se entiende como "aprendizaje colaborativo" y las dimensiones propias de este tipo de aprendizaje, es importante señalar los elementos que intervienen en el aprendizaje colaborativo mediado por tecnología para lograr sus objetivos.

1. Interdependencia positiva: Bravo Santos (2002) menciona primero Interdependencia positiva, ésta se refiere a depender de los compañeros de trabajo para realizar objetivos, tareas, recursos, roles y recompensas, esto puede darse en interacciones verbales cara a cara y no verbales (como en el caso de la comunicación escrita en red). Lucero (2003), habla de la interdependencia positiva como el elemento central del CSL, ya que los miembros del grupo deben necesitarse uno al otro y confiar en las capacidades y entendimiento de los demás, la autora concuerda con Bravo Santos en que la interdependencia se da en torno a los objetivos, tareas, etc.

2. Contribución individual: Cada miembro del grupo debe asumir íntegramente su tarea y, además, tener los espacios para compartirla con el grupo y recibir sus contribuciones (Lucero, 2003, p.4); a esto, Scagnoli y Norma (2006) agregan que cada uno de los miembros del grupo es responsable por su aporte individual y por la manera que ese aporte contribuye al aprendizaje de todos (p. 2).

3. Habilidades personales y de grupo: La vivencia del grupo debe permitir a cada miembro el desarrollo y potencialización de sus habilidades personales; de igual forma, permitir el crecimiento y la obtención de habilidades grupales como: escucha, participación, liderazgo, coordinación de actividades, seguimiento y evaluación (Lucero, 2003 p.4). Esta habilidad también es llamada colaborativa, y se dice que los estudiantes deben entender que el aprendizaje es social. Deben también estar dispuestos a escuchar las ideas de los demás, a articularlas efectivamente, tienen empatía por los demás y una mente abierta para conciliar con ideas contradictorias u opuestas. Poseen la habilidad para identificar las fortalezas de los demás (Collazos, Guerrero \& Vergara ,2001 p.3).

4. Interacción: Son los procesos complejos de relación interpersonal o intergrupal, entre las personas y los objetos, y de los individuos (o grupos) con los entornos o contextos en los que interactúan (Londoño, 2008 p. 15). 
Tabla 1. Herramientas TIC para el aprendizaje colaborativo.

\begin{tabular}{|c|c|c|c|c|}
\hline Herramienta & Comunicación & $\begin{array}{l}\text { Publicación } \\
\text { de trabajo } \\
\text { en web }\end{array}$ & $\begin{array}{l}\text { Gestión } \\
\text { del trabajo } \\
\text { colaborativo }\end{array}$ & $\begin{array}{l}\text { Búsqueda de } \\
\text { información }\end{array}$ \\
\hline Chat-mensajería instantánea & $\mathrm{X}$ & & & \\
\hline Correo electrónico & $\mathrm{X}$ & & & \\
\hline Foros & $\mathrm{X}$ & & & \\
\hline Video conferencia & $\mathrm{X}$ & & & \\
\hline Wikis & & $\mathrm{X}$ & & \\
\hline Blogs & & $\mathrm{X}$ & & \\
\hline Redes Sociales & $\mathrm{X}$ & $\mathrm{X}$ & & \\
\hline Plataformas LMS & & & $\mathrm{X}$ & \\
\hline Calendarios Compartidos & & & $\mathrm{X}$ & \\
\hline Plataformas de trabajo colaborativo & & & $\mathrm{X}$ & \\
\hline Buscadores & & & & $\mathrm{X}$ \\
\hline
\end{tabular}

Fuente: Elaboración propia con base en Vinagre (2010) y Díaz Barriga \& Morales (2008).

La interacción ocupa un papel primordial en esta investigación. Promoverla permitirá que se vea un verdadero trabajo en conjunto y alentará los esfuerzos de los miembros del grupo, así mismo, facilitará el éxito de cada uno para alcanzar la meta común (Johnson, Johnson \& Smith, 1998, p. 5-16). Las formas de interacción realizadas en una interdependencia positiva son las que influencian los resultados de aprendizaje, el contacto ayuda a realizar el seguimiento y el intercambio entre los diferentes miembros del grupo; el alumno aprende de ese compañero con el que interactúa día a día, o él mismo le puede enseñar, cabe apoyarse y apoyar.

En la medida en que se posean diferentes medios de interacción, el grupo podrá enriquecerse, aumentar sus refuerzos y retroalimentarse (Lucero, 2003, p. 4). Los grupos que fomentan una interacción promotora son los que perciben que sus objetivos se correlacionan con los de sus compañeros, este tipo de interacción promueve la comprensión mutua mediante el diálogo, el apoyo, la ayuda y el elogio. Los miembros del grupo apoyan al compañero que no entiende alguna pregunta o solución a un problema; un punto importante para promoverla es asegurarse que cada uno recibe la ayuda que necesita (Vinagre, 2010, pp. 52-53).

Hay tres pasos según Johnson et al. (1998), para fomentar la interacción que genera apoyo académico y personal en los grupos colaborativos: 1) programar tiempo para que el grupo se reúna, ya que no siempre se da tiempo para que los grupos maduren y se desarrollen; 2) generar interdependencia positiva para que se genere un compromiso mutuo para llegar a la meta común; 3) fomentar la interacción promotora y celebrarla cuando se lleve a cabo (p. 5-16).

Una de las bondades más importantes que tiene la interacción, es que proporciona ayuda y asistencia efectiva al intercambiar recursos necesarios (información y materiales) para procesar la información de manera más eficaz, además, la retroalimentación que reciben los alumnos posterior a su desempeño los ayuda a tener una mejor comprensión y a resolver de mejor forma los problemas, lo que hace que los esfuerzos de todos se vean beneficiados por decisiones tomadas de miembros trabajando juntos de forma real (Johnson et al., 1998).

Es importante que los grupos que se organizan para trabajar en CSL cumplan las características propias del aprendizaje colaborativo (que incluyen la interacción), ya que las redes computacionales o los softwares no harán que se dé automáticamente el aprendizaje, aquí es donde entra la importancia de una correcta planeación por parte del profesor y fomentar las habilidades necesarias para tener aprendizaje colaborativo. Además, en el caso específico del CSL, debe conjuntar el uso correcto de las redes y la computación.

En el ciberespacio se relacionan personas con distintas lenguas, culturas y características (psicológicas, cognitivas, ideológicas y sociales). Para que logren una interacción, establecen lenguajes y protocolos (reglas o normas) propios 
de este ciberespacio y las computadoras que permitan el desarrollo de las habilidades (Londoño, 2008 p. 13).

Se puede ver que son muchas las características que requiere un grupo para pasar de ser tradicional, que solo trabaja de manera individual para lograr metas también individuales, a pasar a ser colaborativo, el cual trabaja por metas comunes que, a la par, desarrollará las habilidades personales. Lo que se buscó en esta primera parte de la investigación doctoral fue conocer, específicamente, cómo se desarrolla la interacción y qué herramientas tecnológicas se utilizan para desarrollarla en el trabajo en equipo de los alumnos de la Licenciatura en Comunicación y Periodismo de la Universidad Autónoma de Querétaro. Para ello, como primer paso, se realizaron seis entrevistas semiestructuradas a profesores que permitieron conocer un poco más a profundidad estas dimensiones.

Entre los resultados se encontró que a los alumnos les cuesta tener una interacción profunda con sus compañeros, ya que sigue imperando un esquema de comunicación donde el profesor es el centro del conocimiento. La mayoría de los profesores coincidió en que existen dos tipos de alumnos, los que son muy aplicados y controlan la interacción en el grupo y, por otro lado, los alumnos que aprovechan el cobijo del trabajo en equipo para realizar la menor actividad posible, lo que deja ver que la interacción no se da de una forma distribuida. Por otro parte, se conoció que Facebook y WhatsApp son las TIC que más utilizan los profesores para apoyar el proceso del trabajo en equipo, sobre todo la red social Facebook que, además de ser utilizada para comunicar, se usa como un repositorio para compartir información de las temáticas de la clase.

\section{Metodología}

Se optó por trabajar con la entrevista semiestructurada para saber los conocimientos y experiencias que tienen los profesores al trabajar en equipos, ya que las respuestas obtenidas, no solo narraban lo que sucede con sus alumnos al trabajar de esta forma, sino que dejaron en claro su concepción sobre el trabajo colaborativo como un método de aprendizaje y sus ideas sobre los papeles que desempeñan sus alumnos al trabajar de esta manera.
Para este artículo, de las categorías revisadas se retomaron dos:

1. La interacción que se genera entre los estudiantes (procesos complejos de relación interpersonal o intergrupal, entre las personas y los objetos, y de los individuos -o grupos- con los entornos o contextos en los que interactúan) (Londoño, 2008 p. 15).

2. Se exploró qué tipo de herramientas tecnológicas usan los profesores y alumnos para comunicarse de manera colaborativa; estas permiten que la comunicación se realice de forma síncrona o asíncrona y su uso lleva a apoyar las discusiones grupales para intercambiar información, resolver problemas o definir formas de trabajo (Tabla 1).

El uso de estas categorías se justifica por lo mencionado por Flick (2007) quien sostiene que estas ayudan a hacer más explícito lo que el entrevistado sabe, además, el entrevistado puede aceptar o rechazar estas ideas confirmando o poniendo en duda los supuestos de los que se parte, lo que ayuda a contrastar los supuestos teóricos con la práctica de los docentes en el aula. En este caso, se trabajó bajo los supuestos de un proceso de inducción analítica poniendo a prueba las proposiciones que hay sobre el desarrollo de la vida social (en este caso del trabajo colaborativo en clase), para ello se utiliza "La inducción analítica que es un procedimiento para verificar teorías y proposiciones basado en datos cualitativos" (Glaser y Strauss, 1967; citados en Taylor y Bogdan, 1987, p.156).

La idea de esta fase de la investigación doctoral fue saber cómo se da en la realidad la interacción en el trabajo colaborativo en estos grupos de estudiantes universitarios y contrastar con las características que deben presentar los grupos considerados colaborativos. Con lo anterior, se puede tener una idea de qué les hace falta a estos grupos tradicionales para que su interacción se considere que tiene realmente rasgos del aprendizaje colaborativo.

Se tomaron como base seis entrevistas semiestructuradas con profesores de la Licenciatura en Comunicación y Periodismo de la Universidad Autónoma de Querétaro, los cuales, debido a la naturaleza de sus materias, trabajan de forma colaborativa o en equipo; los semestres en los que imparten clase son diversos y van desde el cuarto semestre hasta el sexto del plan de estudios 2004. La intención del 
estudio era conocer de qué forma se da la interacción al trabajar por equipo o de manera colaborativa, qué tipo de tecnología usan sus alumnos para realizar el trabajo en equipo, y cómo se da la interacción con estas herramientas.

Para ello se buscó conocer cómo describen los profesores la forma en que sus alumnos trabajan en equipo y cómo se desarrolla la interacción entre ellos, ya sea presencial o a distancia, puesto que la manera en que definen la interacción de sus alumnos brinda pistas sobre cómo sucede este proceso, desde la voz de sus protagonistas.

\section{Resultados}

La dimensión de la interacción en el aprendizaje colaborativo tiene que ver con la relación que se da entre los alumnos al momento de colaborar, es decir, la forma en que los alumnos se comunican entre ellos y con los profesores. Además, esta dimensión incluye la noción de que el alumno enseña a sus compañeros durante el proceso social de aprendizaje y que él, a su vez, aprenda de ellos. En cuanto a la comunicación, esta dimensión contempla la retroalimentación constante, es decir, un modelo de comunicación más democrático donde todos participan y todos se escuchan, donde las decisiones se toman en conjunto con base en el diálogo que se establece.

En el análisis general de esta dimensión se pudo observar que los profesores mencionaron una dificultad para establecer una comunicación constante entre los alumnos durante el proceso de trabajo en equipo; manifestaron que constantemente tienen que estar recordándoles que deben dialogar con sus compañeros; la mayoría dice que están más acostumbrados a buscar al profesor para que guíe la comunicación durante el proceso y sea él quien los auxilie en la toma de decisiones que requieren estos procesos de trabajo.

Otra problemática aludida por los profesores, son los problemas personales que tienen entre los alumnos, previo a llegar a la clase, ya que en muchas ocasiones, al no poder escoger a sus compañeros de equipo, manifiestan un descontento por tener que trabajar con ciertos integrantes. En este caso los maestros apelan a la idea de que en el campo laboral los estudiantes no podrán escoger con quién trabajar, por lo que deben aprender a comunicarse e interactuar con todo tipo de personas, sin embargo, ninguno dijo tener alguna estrategia para apoyar a los alumnos en la resolución de los conflictos que pueden surgir a partir de la interacción en el trabajo en equipo.

Un punto importante que surgió tiene que ver con los papeles que juegan los alumnos en el trabajo grupal, todos los profesores hablaron de dos tipos de alumnos; los primeros son los que están muy interesados por el trabajo y los que normalmente lideran los equipos, estos llegan a acaparar el diálogo, son impositivos en las tomas de decisiones y, aunque pretenden escuchar a sus compañeros, terminan tomando las decisiones importantes, lo que en este caso no permitiría el desarrollo de un diálogo que consienta construir en conjunto la solución de los problemas a los que se enfrentan.

Por otro lado, mencionaron a los alumnos que no les interesa mucho trabajar con sus compañeros, los profesores dijeron que este tipo de alumnos se aprovecha de la cobertura que da el trabajo en equipo y tratan de trabajar lo menos posible, se distraen y salen del salón cada vez que pueden; si están con sus compañeros no generan diálogo, simplemente obedecen las órdenes de quienes lideran y, en la mayoría de los casos, buscan hacer actividades de poca importancia con el fin de cumplir, aun cuando realmente no se están involucrando demasiado en el proceso del trabajo en equipo.

Con relación a la problemática de los dos tipos de alumnos, los docentes no mencionaron alguna estrategia para que los que trabajan menos se integren, y los que trabajan más aprendan a escuchar y delegar responsabilidades: los maestros parecen tomar este tipo de actitudes como algo natural del trabajo en equipo, incluso se llegó a mencionar que esta es una de las desventajas de trabajar en equipo, lo ven como algo inevitable. La única respuesta a este fenómeno es sancionar a través de las formas de evaluación, recompensar a quien trabaja y castigar a quien no lo hace.

En cuanto al uso de la tecnología para la interacción en el trabajo en equipo, prácticamente todos los profesores utilizan la red social Facebook para mantenerse en contacto fuera del aula con sus alumnos; el tipo de interacción que hacen en esta red principalmente es compartir contenidos, dar avisos con respecto de la clase y subir el trabajo que generan los alumnos durante el curso.

Otra plataforma que usan para la interacción es WhatsApp. Resulta interesante ver que todos la usan de manera similar, 
les sirve para comunicarse con los líderes o los jefes de grupo y para dar avisos que por su naturaleza son urgentes, por lo que los mensajes en esta aplicación no van dirigidos a todos los alumnos del grupo.

El uso del correo electrónico es poco mencionado. Cuando lo llegan a utilizar es para generar mensajes que son muy formales y que requieren de una contestación más pensada y meditada. Cabe destacar que, de los profesores, solo uno utiliza el correo electrónico para comunicarse con sus alumnos fuera del aula y, en este caso, la comunicación se da con el líder del equipo o el representante del grupo y es básicamente para compartirle los materiales de la clase; toda la demás interacción se da cara a cara o en el cubículo del profesor.

Uno de los profesores fue muy enfático al asegurar que la tecnología le ha traído muchas dificultades a su grupo para el proceso de trabajo en equipo que están elaborando este semestre, pues para este proyecto los alumnos deben trabajar en colaboración con un grupo de un turno distinto. Menciona que la comunicación a través de redes sociales ha sido caótica, que hay muchos malos entendidos, que se malinterpretan los mensajes, que existe poca participación por parte de todos los alumnos y comenta que la solución más viable fue generar interacciones cara a cara, donde pudo ver que los alumnos se pusieron de acuerdo de una manera más sencilla y lograron solucionar sus problemas. Para él, en muchas ocasiones, el uso de la tecnología llega a hacer más difícil el proceso, por lo que considera que hace falta capacitación para alumnos y maestros en cuanto a sus habilidades digitales.

Algunos profesores manifiestan que, conforme avanza el trabajo en equipo, los alumnos afinan su manera de interactuar, ya que en un principio les tienen que estar recordando constantemente que se comuniquen para que el trabajo realmente sea en equipo; conforme se avanza en el proceso, comienzan a comunicarse más y crean una forma de interacción propia para avanzar en las dificultades del proyecto.

Sin embargo, algunos maestros aseguran que los estudiantes solo simulan resolver las problemáticas a las que se enfrentan y que lo que en realidad hacen es evadir y dar la vuelta a los problemas, subdividiéndose las tareas y acordando que cada quien aporte una parte del trabajo, sin tener que llegar a discutir o negociar cómo hacerlo; si a esto se agrega la problemática ya comentada anteriormente sobre los liderazgos controladores, se puede ver en algunos casos un tipo de interacción que se centraliza en algunas personas y que no permite el diálogo, la escucha, la renuncia a las ideas propias por el bien común, y que, por otro lado, fomenta la imposición, la simulación y los procesos de diálogo que no involucran a la mayoría de los participantes.

Finalmente, algunos profesores manifiestan que la dificultad de la interacción en el trabajo en equipo viene de una formación cultural y social propia del país; manifiestan que se ha formado a los alumnos esperando que un líder indique exactamente qué hacer y guíe en la solución de problemas; comentan que esta formación lleva a tener vicios como la impuntualidad, la falta de flexibilidad para renunciar a las ideas propias, la carencia de habilidades para resolver problemas, la poca disposición a trabajar con otros; vicios que obstaculizan el flujo de trabajo por equipos. Cabe señalar, de nuevo, que los profesores no comentaron tener alguna estrategia para remediar este tipo de manifestaciones que observan cuando sus alumnos interactúan.

\section{Discusión}

Uno de los primeros resultados que comentan todos los profesores entrevistados, es la dificultad para que sus alumnos tengan una interacción y que juntos puedan resolver problemas y ayudarse mutuamente para realizar la tarea y de esa forma aprender juntos.

Esta dificultad se presenta, entre otras cosas, porque los profesores no guían los procesos de interacción, provocando que los alumnos lleguen a la meta haciendo un esfuerzo individual o cobijando la pereza de otros, pues, como se decía antes, existen en los grupos, según los maestros, los alumnos que contribuyen con la tarea y los alumnos que no lo hacen. Al no regular la interacción y la participación entre estos dos tipos de estudiantes, el proceso de la interacción no es desarrollado, por lo que los estudiantes no tienen necesidad de interactuar para resolver la tarea propuesta.

Los problemas personales que tienen, previo a trabajar en actividades colaborativas o del grupo, es otra de las dificultades que fue mencionada por los docentes, ya que dicen que los alumnos no quieren trabajar con ciertos compañeros que no les caen bien. La solución que plantean los profesores es concientizarlos de que no siempre van a trabajar con quién 
quieren; sin embargo, de nuevo se nota que no se fomenta un proceso de interacción entre los alumnos, en el cual se busque que sean ellos los que, a partir de las relaciones que hagan, solucionen sus problemas y busquen la forma de cumplir la meta de manera conjunta. Se puede ver que la comunicación que plantean los profesores en sus actividades grupales sigue centrada en las instrucciones que ellos generan, lo que no permite una interacción entre iguales y le resta fuerza a las relaciones entre alumnos.

Como se mencionó, un dato interesante es la tipología de los alumnos que trabajan de forma colaborativa, ya que casi todos los profesores señalaron dos tipos de alumnos, los que son muy comprometidos con la tarea a realizar, pero que en muchos de los casos llegan a ser impositivos, poco tolerantes y en ellos se centraliza la comunicación del equipo, normalmente ellos deciden las soluciones ante los problemas que se presentan. Por otro lado, hablaron de los alumnos que, ante el cobijo del trabajo en equipo, no participan, no trabajan, hacen el mínimo esfuerzo y, en la mayoría de los casos, se contentan con obedecer a sus compañeros más comprometidos.

Cabe destacar que los profesores ven esto como algo natural y en ningún momento plantean alguna alternativa para que esto funcione de una manera distinta, lo que llevaría a pensar que a pesar de que existe trabajo en equipo, no se puede hablar de grupos colaborativos, ya que no existe una meta común por las que todos trabajen ni una solución de problemas en forma conjunta. Se puede decir que la interacción no es la deseada para un grupo colaborativo, puesto que hay docentes que, incluso, se atreven a decir que los alumnos no solucionan los problemas sino que les dan la vuelta y trabajan de forma individual para llegar a la meta.

Una manera de generar la participación más equitativa de los estudiantes, es hacer que las actividades a realizar generen una interdependencia positiva, que todos dependan de todos para llevar a cabo la tarea y llegar a la meta común.

El uso de la tecnología para colaborar es un punto clave en la investigación en curso aquí presentada. Se pudo ver que, a pesar de haber distintas herramientas que pueden enriquecer el proceso colaborativo a distancia, la mayoría de los profesores utilizan dos tecnologías cuyo uso primordial es la comunicación con los alumnos fuera del aula, y, por otro lado, la función de compartir archivos tanto de autores externos como de los propios alumnos. También, se notó que solo uno de los profesores hace un uso constante de la tecnología para, a través de ella, fomentar la colaboración.

La postura ante el uso de la tecnología es dividida, ya que la mayoría de los profesores, aunque la usan, no manifiestan que sea algo vital para el desarrollo de su clase; incluso uno de los profesores comentó que, en el proceso actual de su clase, la tecnología ha hecho que se complique más la interacción entre sus alumnos. La solución que plantearon ante esta disyuntiva fue generar interacción cara a cara y de esa forma se solucionó el problema.

Es posible ver en este caso que, por un lado, hay un conocimiento limitado de los tipos de tecnologías que pueden enriquecer el proceso colaborativo, pues los profesores usan tecnologías ya muy conocidas (Facebook y WhatsApp) y solo uno de ellos explora distintas alternativas educativas; $y$ por otro, se pudo ver el problema de la falta de desarrollo de trabajo colaborativo utilizando las bases mínimas para que esto se dé. Por lo que el utilizar tecnología hace que el proceso colaborativo sea aún más complicado, ya que, si desde el principio no se plantean las bases para el trabajo en grupo, usar la tecnología solamente hará que las problemáticas aumenten, entonces se puede pensar que existe una falta de capacitación en el uso de métodos colaborativos y de uso de la tecnología educativa.

Es interesante conocer la concepción que tienen los profesores sobre el trabajo colaborativo, puesto que manifiestan que las dificultades que presentan sus alumnos para hacer un verdadero trabajo colaborativo provienen de su formación social y cultural: comentan que es difícil desarraigar prácticas como el individualismo y la falta de escucha y, como se mencionaba, ven este proceso como algo natural, por lo que es importante acercar a los profesores los principios de los métodos colaborativos y del uso de la tecnología para que puedan, por un lado, entender lo que pasa en su clase; y, por el otro, ver que es necesario apoyar el desarrollo de la interacción y fomentar las relaciones de aprendizaje entre los alumnos cuando se trabaja en equipo.

Al saber que hay una forma de trabajo ya establecida y que esta puede ayudar a que los procesos colaborativos mejoren, se puede ayudar a generar un cambio en la idea de que este tipo de labor siempre tendrá dificultades, y que sus alumnos no son capaces de generar un verdadero trabajo colaborativo. 


\section{Conclusiones}

Del análisis de los resultados y de la discusión realizada se puede llegar a las siguientes conclusiones:

1. La interacción para trabajar en equipo es un problema, pues simulan trabajar entre ellos cuando realmente cada quien aporta su parte sin interactuar con el compañero;

2. Predominan dos tipos de alumnos: el líder, que trabaja durante todo el proceso y no toma en cuenta la opinión de los demás, y el que se cobija en el trabajo de los otros para hacer lo menos posible;

3. La tecnología para el trabajo en equipo es utilizada, pero los profesores comentaron que pueden trabajar sin ella;

4. WhatsApp y Facebook son las tecnologías más utilizadas, principalmente para comunicar y como repositorio de archivos sin explotar su potencial de red social y;

5. Las dificultades del trabajo colaborativo provienen de la formación social y cultural del alumno, imperante en el país.

Se puede observar que los problemas existentes a la hora de trabajar vienen derivados, en muchos de los casos, de no estructurar y coordinar las interacciones para construir la solución de un problema en conjunto (Stahl et al., 2006). Esto se debe a que los profesores no mencionaron regular los papeles de liderazgo ni la comunicación, que se puede ver entorpecida por el tipo de relación que generan los alumnos. Como menciona Bravo (2002), se debe generar interdependencia positiva: cada alumno necesita del otro para realizar la tarea, y esto solo se puede dar con una planificación y evaluación de la actividad colaborativa.

Además, no hay una intervención en los problemas que se generan entre los alumnos, estos son fuente de diálogos deficientes ya que, como menciona Vinagre (2010), se debe fomentar una comunicación donde exista la comprensión mutua mediante el diálogo, el apoyo, la ayuda y el elogio.

Por otro lado, se puede ver un uso limitado de la tecnología para la comunicación, ya que las herramientas tecnológicas que utilizan los profesores tienen una naturaleza social. Como se pudo observar, las redes sociales y los mensajes instantáneos sirven para generar interacción entre los alumnos y, cuando es requerido, con el docente (Tabla 1); sin embargo, el uso que se les da a estas herramientas es limitado (solo para mandar mensajes cortos y enviar archivos). Hace falta que se les den a conocer las posibilidades educativas de las TIC y ayudarlos a establecer una relación con los fundamentos didácticos de la colaboración.

Cuando se menciona que las redes sociales pueden generar más problemas para la colaboración, se observa la necesidad de preparar a los profesores en el campo del aprendizaje colaborativo mediado por tecnología (CSL). Esta perspectiva conjuga los saberes del aprendizaje colaborativo con el uso de redes computacionales (Londoño, 2008). Al no enseñar las formas de colaboración específicas que tiene la red, dificultan la entrada plena de los alumnos a un espacio cibercultural donde se amplifican los problemas para trabajar colaborativamente de manera presencial.

Esta información sirve como base para conocer las necesidades de formación que requieren los profesores para que la estrategia colaborativa mediada por tecnología que se diseñará, las contemple, y apoye en el proceso de creación de grupos colaborativos efectivos en la Licenciatura de Comunicación y Periodismo de la Universidad Autónoma de Querétaro; si bien, este no es un problema nuevo, sí funciona a manera de contexto, pues permite observar que, a través de la experiencia de los profesores, se trabaja en equipo sin cumplir con las características que se requieren para tener un verdadero trabajo colaborativo y gozar de los beneficios que esta metodología ofrece.

\section{Referencias}

Bravo Santos, C. (2002) Un sistema de soporte al aprendizaje colaborativo del diseño domótico mediante herramientas de modelado y simulación. Tesis de Doctorado: Universidad de Castilla. La Mancha, España. Recuperado de https://ruidera.uclm.es/xmlui/handle/105 78/905

Collazos C., Guerrero, L. \& Vergara A. (2001) Aprendizaje colaborativo: un cambio en el rol del profesor. Actas del Tercer Congreso de Educación Superior en Computación, Punta Arenas, Chile, noviembre, 2001.Recuperado de https://users.dcc.uchile.cl/ luguerr e/papers/CESC-01.pdf

Díaz Barriga, F. \& Morales Ramírez, L. (2008). Aprendizaje colaborativo en entornos virtuales: 
un modelo de diseño instruccional para la formación profesional continua (e-version). Tecnología y Comunicación Educativas, 22-23(1).4-25 Recuperado de http://gc.initelabs.com/recursos/files/r161r/w2382 8w/aprendizaje_colaborativo_EVA.pdf

Flick, U. (2007). Una introducción a la investigación cualitativa. España. Morata SL.

González, R. M. G., González, L. G., de la Cruz, N. M., Fuentes, M. G. L., Aguirre, E. I. R., \& González, E. V. (2012). Acercamiento epistemológico a la teoría del aprendizaje colaborativo. Apertura, 4(2), 156-169. Recuperado de http://www.udgvirtual.udg.mx/apertura/i ndex.php/apertura/article/view/325

Johnson, D. W., Johnson, R. T., y Smith, K. A. (1998). Active learning: Cooperation in the college classroom. Interaction Book Company, 7208 Cornelia Drive, Edina, MN 55435.

Londoño, G. (2008). Aprendizaje colaborativo presencial, aprendizaje colaborativo mediado por computador e interacción: Aclaraciones, aportes y evidencias. Revista $Q, 2(4)$. Recuperado de https://search.proquest.com/op enview/1cbb6997615baf4906eb8e89e06dd707/1?pqorigsite $=$ gscholar $\& \mathrm{cbl}=1666340$

Lucero, M. M. (2003). Entre el trabajo colaborativo y el aprendizaje colaborativo. Revista iberoamericana de Educación, 33(1), 1-21. Recuperado de https://rieoei .org/RIE/article/view/2923/3847

Scagnoli, I., \& Norma, M. (2006). El aprendizaje colaborativo en cursos a distancia. Investigación y ciencia, 14(36). Recuperado de http://www.redalyc.org/html/674/67403 608/

Stahl, G., Koschmann, T. \& Suthers, D. Aprendizaje Colaborativo apoyado por computador: Una perspectiva histórica. Cambridge: RK Sawyer. Recuperado de http://gerrystahl.net/cscl/cscl_spanish.h tm

Taylor, S. J., \& Bogdan, R. (1987). Introducción a los métodos cualitativos de investigación (Vol. 1). Barcelona: Paidós.

Vinagre, M. (2010). Teoría y práctica del aprendizaje colaborativo asistido por ordenador. Madrid: Editorial Síntesis. 